This paper is published as:

Tang, Z, McCabe, BY, 2007, "Developing Complete Conditional Probability Tables from Fractional Data for Bayesian Belief Networks" Journal for Computing in Civil Engineering, 21(4):265-276

\title{
DEVELOPING COMPLETE CONDITIONAL PROBABILITY TABLES FROM FRACTIONAL DATA FOR BAYESIAN BELIEF NETWORKS
}

\author{
Zhong Tang ${ }^{1}$ and Brenda McCabe ${ }^{2}$
}

Key words: Knowledge-based systems, Artificial intelligence, Bayesian analysis, Airport construction, Probabilistic models, Data collection

\begin{abstract}
Bayesian belief network (BBN) can be a powerful tool in decision making processes.

Development of a BBN requires data or expert knowledge to assist in determining the structure and probabilistic parameters in the model. As data are seldom available in the engineering decision making domain, a major barrier in using domain experts is that they are often required to supply a huge and intractable number of probabilities. Techniques for using fractional data to develop complete conditional probability tables were examined. The results showed good predictability of the missing data in a linear domain by the piecewise representation method. By using piecewise representation, the number of probabilities to be elicited for a binary child node with $k$ binary parent nodes is now $2 k$ rather than $2^{k}$.
\end{abstract}

\section{INTRODUCTION}

Bayesian belief networks (BBN) refer to a method in artificial intelligence associated with the Reverend Thomas Bayes' (c. 1702-1761) theorem on conditional probability. BBN are directed acyclic graphs (DAGs) in which the nodes represent variables, and the arcs signify dependence between the linked variables. The strength of these influences is expressed by conditional probabilities (Pearl 1988). BBN provide a knowledge base to encode the structure of relevancies as well as probabilistic relationships. Therefore, BBN can be a powerful tool in decision making processes.

With the maturity of the theories and algorithms of BBN in the 1990s, BBN have been applied successfully in various fields. For example, the applications of BBN in Microsoft's products including the Answer Wizard of Office 95, the Office Assistant (the bouncy paperclip) of Office 97, and over 30 Technical Support Troubleshooters (Heckerman et. al. 1995). There are also applications in bioinformatics and medical informatics (Di Bacco et. al. 2004; Husmeier et. al. 2005); in interplanetary probes and deep space explorations (Stutz et. al., 1998); and in civil and

\footnotetext{
1 Zhong Tang, Lecturer, Department of Civil Engineering, Curtin University of Technology, GPO Box U1987, Perth, 6845, Australia Email: Z.Tang@curtin.edu.au

${ }^{2}$ Brenda McCabe, Associate Professor, Department of Civil Engineering, University of Toronto, Toronto, ON, Canada M5S 1A4 Email: Brenda.mccabe@utoronto.ca
} 
construction engineering (McCabe et. al. 1998; Nasir et. al. 2003). Situations that are less suited to BBN may include those in which non-probabilistic responses are required, or where graphical representation of the relationships between domain variables is inappropriate.

A belief network is symmetric in that a variable may be instantiated as an input variable or may be evaluated as an output of the model, enabling a model to provide both diagnostic and decision support simultaneously. The flexibility to utilize variables as either input or output without redesigning the system is uncommon in other forms of artificial intelligence (Al) representation, such as rule-based expert systems or artificial neural networks.

Maintenance of a knowledge base requires that there exist an efficient method of updating the model. The graphical nature of belief networks permits variables and arcs to be modified without significantly affecting the remainder of the network because modifications to the network may be isolated. Probabilistic relationships and values that require examination include only those directly connected to the graphical element(s) being added, removed, or modified.

Figure 1 shows a simple BBN, referred to as a naïve BBN, where there is just one child node and multiple parent nodes. $\mathrm{P} 1$ to $\mathrm{Pk}$ are called the parents of $\mathrm{C}$, and $\mathrm{P} 1$ to $\mathrm{Pk}$ are orphans in that they have no parents. Formally, the parents of $C_{i}$ are those variables judged to have a direct influence on $\mathrm{C}_{\mathrm{i}}$. The degree of influence is quantified via conditional probability tables (CPT) embedded in the networks. The number of probabilities required for each node is shown in Eq. ( 1 ).

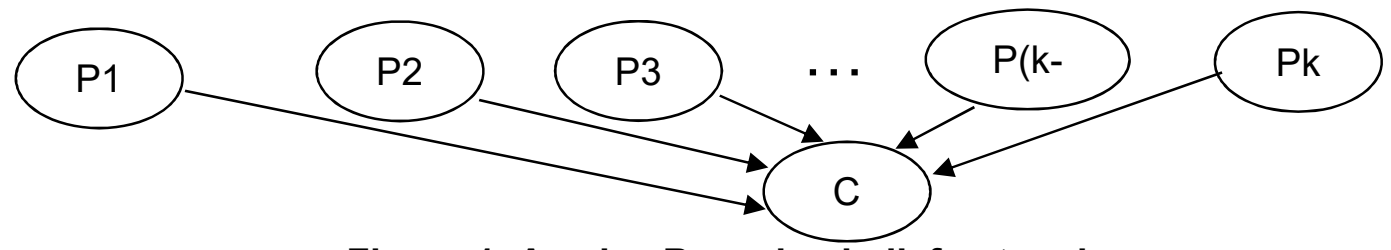

Figure 1. A naive Bayesian belief network

$$
N P=(m-1) \prod_{i=1}^{k} n_{i}
$$

where $N P$ is the number of probabilities of the child node, $m$ is the number of states of the child node, and $n$ is the number of states of parent node $i=1 \ldots k$. If a node has no parents, the product term drops from the equation (it does not take the value of zero) and only the prior probabilities $(m-1)$ are needed. In its simplest form with all nodes binary, $N P=2^{k}$. Therefore, if $k=3, N P=8$; but if $k=8, N P=256$; the number of probabilities required increases exponentially with the number of parent nodes. More detailed information on BBN can be found in Pearl (1988), Heckerman (1995) and Tang \& McCabe (2002).

The probabilistic information for the learning of the BBN is available from various sources, which includes statistical data, literature, and human experts (Druzdzel and Van der Gaag 2000). Sarkar and Murthy (1996) presented a technique to construct efficient BBN structures for application areas where large amounts of data are available and information on the ordering of the variables can be obtained from domain experts. They used the I-Divergence measure for evaluating different approximations and discussed how the information obtained from experts leads to improving the efficiency of the technique to find the best network structure. In many areas, however, historical and system data are not available, and elicitation of the knowledge from domain experts provides the foundation for the learning of the BBN. Unfortunately, the elicitation of prior beliefs is a topic that has received relatively little attention in the Bayesian 
literature (O'Hagan 1998) although the elicitation of probabilities is often a major obstacle in building BBN (Van der Gaag et al. 1999).

As shown in Eq. ( 1 ), the number of probabilities required from the domain expert increases dramatically when the network becomes complex and interconnected. The requirement of a large number of probabilities may result in 1) a domain expert not being available or willing to provide all probabilities due to time constraints, or 2) a situation where providing the huge quantity of probabilities in a consistent way becomes an intractable task for a domain expert. To overcome these obstacles, an effective and efficient method for knowledge elicitation from domain experts is needed. Therefore, this research focused on the development of a method for using fractional or incomplete data to estimate the whole domain, thereby facilitating and expediting the process of knowledge elicitation. The context in which this was done is engineering management decision making.

\section{EXPERIMENTAL DESIGN AND KNOWLEDGE ELICITATION}

Airport development projects, which exhibit complexity in decision making, were chosen as the primary knowledge elicitation domain. An ongoing airport development project was closely observed to obtain knowledge and understanding of the complexity of airport development. Experts from this project and from other major Canadian airports were invited to take part in the research.

In airport development, one critical decision relates to the construction of a passenger building (PB). In a brown field development, the airport authority must decide whether to renovate the existing building or construct a new one. Six critical factors influencing this decision were identified, and are defined in Table 1. Based on the relationships between these factors, a naïve BBN was developed as shown in Figure 2. Capital letters are used to identify the nodes; lower case letters are used to indicate the states of the nodes. For example, $(P B=n)$ means the Passenger Building node has a state of "new". All nodes are binary.

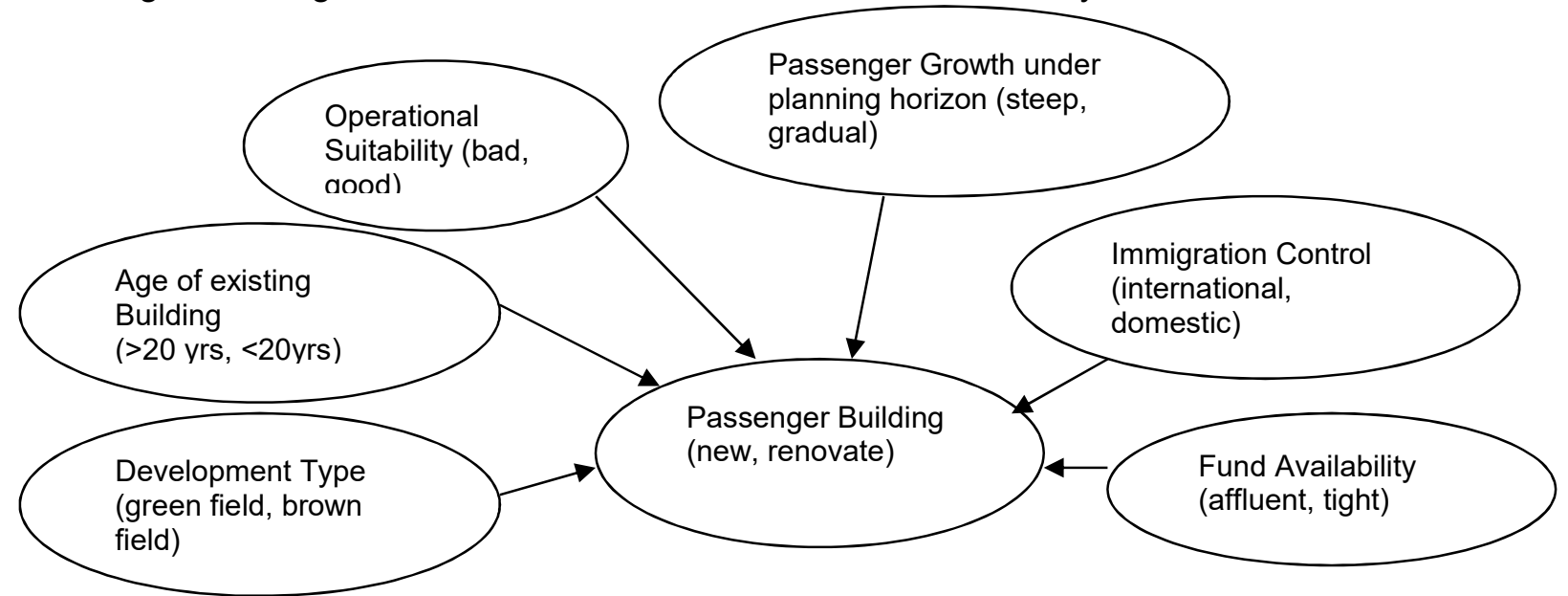

Figure 2. A Bayesian belief network for passenger building construction

When the Development Type is green field $(D T=g)$, the states of other factors will have little effect on the decision of constructing a new passenger building $(P B=n)$ because no building exists for renovation. Factors like OS and $A B$ will drop out under $(D T=g)$, other factors will be overshadowed by $(D T=g)$ in the decision making. Therefore, $(D T=g)$ is referred to as a dominant factor. When a dominant factor exists, the outcome of decision making will be controlled by that 
factor and will be insensitive to the combination of the states of other factors. As a result of recognizing a dominant factor in this $\mathrm{BBN}$, knowledge elicitation from domain experts will be focused on the scenarios when the dominant factor is off, which reduces to half the probabilities needed from the domain experts. Therefore, it is important to identify if dominant factors exist in the BBN before conditional probability tables are elicited from domain experts. The issue is discussed further in a later section of this paper.

Table 1. Variables in airport development decision making

\begin{tabular}{|c|c|c|c|c|c|}
\hline \multicolumn{2}{|r|}{ Variable } & \multicolumn{2}{|r|}{ Favorable state } & \multicolumn{2}{|r|}{ Unfavorable state } \\
\hline PB & Passenger Building & $\mathrm{n}$ & construct a new building & $r$ & renovate existing bldg \\
\hline DT & Development Type & g & green field development & b & brown field development \\
\hline$A B$ & Age of existing Building & $>20$ & more than 20 years old & $<20$ & less than 20 years old \\
\hline OS & Operational Suitability & b & bad condition & g & good condition \\
\hline PG & Passenger Growth & s & increase steeply & g & increase gradually \\
\hline IC & Immigration Control & i & domestic and international & d & domestic only \\
\hline FA & Fund Availability & a & affluent funds & $\mathrm{t}$ & tight budget \\
\hline
\end{tabular}

When the development type is brown field $(D T=b)$, the states of the other 5 factors will have an effect on the decision of constructing a new passenger building $(P B=n)$. For example, the choice of constructing a new passenger building $(P B=n)$ under the condition that the operational suitability of the existing building is bad $(O S=b)$ is more probable than that under the condition that the operational suitability of the existing building is good $(O S=g)$. Therefore, $(O S=b)$ is considered a favorable state on the choice of $(P B=n)$, and $(O S=g)$ is considered an unfavorable state on the choice of $(P B=n)$. In this research, $(P B=n)$ is the baseline choice, the favorable and unfavorable states of other factors in the network are defined with respect to the choice of $(P B=n)$. For the 5 parents that affect $(P B=n)$ given that $(D T=b)$, there exist $32\left(2^{5}\right)$ combinations or scenarios of parent states, each requiring a conditional probability from the domain experts.

To ease the burden for domain experts in providing large amounts of conditional probabilities, experiments were designed to look at the feasibility of asking one domain expert to provide fractional data and then to use interpolation based on certain rules to acquire the whole domain. The experimental design is shown in Figure 3. Data collection consisted of 3 well-spaced (several weeks) rounds with seven experts. The experts were asked to provide conditional probabilities for various scenarios in each round, where round 1 had 6 scenarios, round 2 had 14 and round 3 contained the full set of 32 conditional probabilities that covered the entire domain. Through the comparison of the feedback from rounds 1, 2 and 3 of each domain expert, the consistency between the rounds for each expert was studied and the potential for predicting the whole domain by interpolating fractional data from a domain expert was examined. 
Problem: Knowledge elicitation in probabilistic knowledge models is difficult for nodes with many parents. Therefore, a method for reducing the number of probabilities from experts is required.

Experimental Methodology: Using incomplete data from one expert to estimate a complete set of probabilities

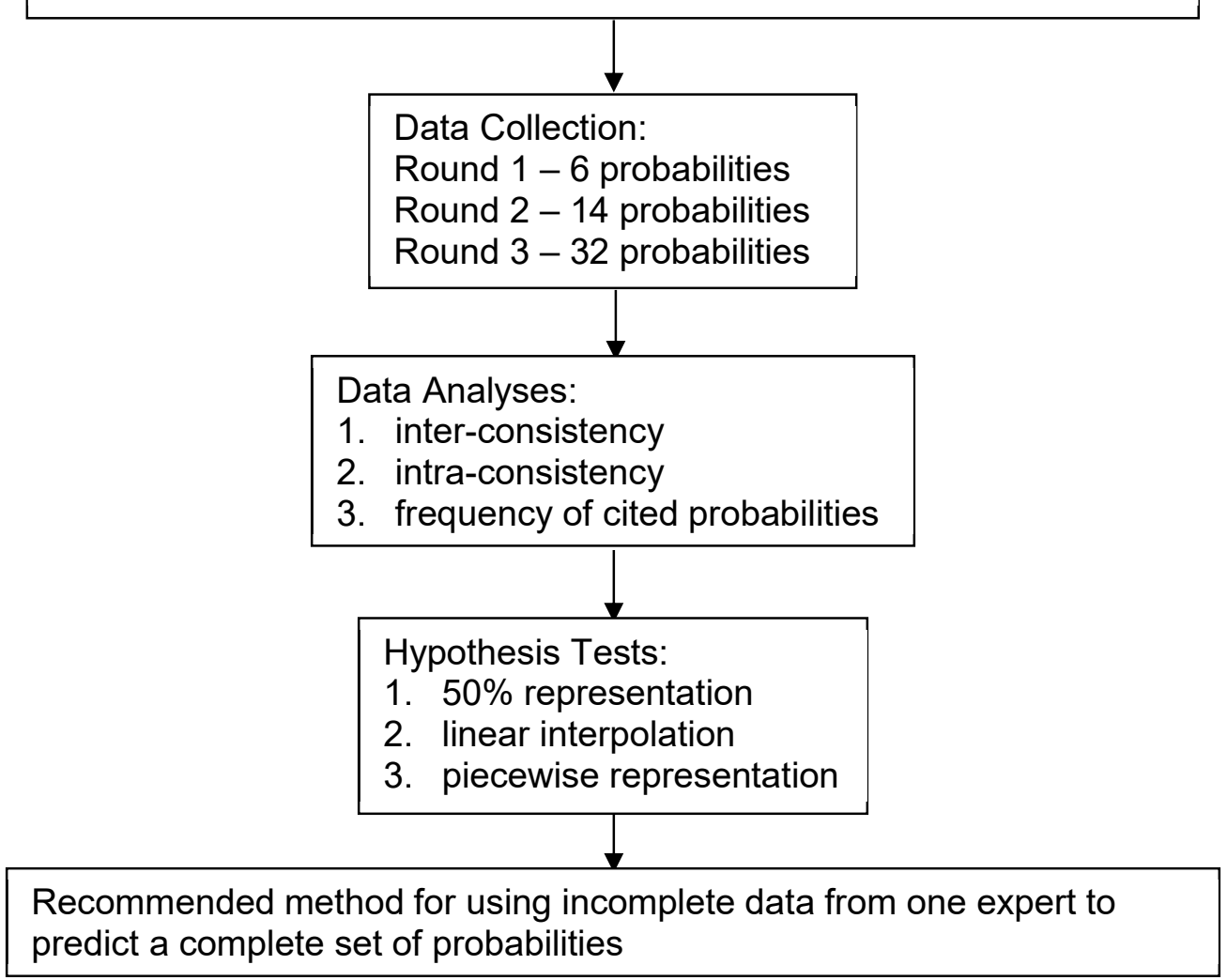

Figure 3. Flowchart of the experimental design

The three rounds were completed as shown in Table 2 . To represent a scenario concisely and facilitate the discussion, the scenario number and the number of unfavorable states on $(P B=n)$ are used in the form of "No.-j". For example, $\operatorname{Pr}(P B=n \mid D T=b, O S=b, P G=s, I C=i, F A=a$, $A B=>20)$ is "c1-0", $\operatorname{Pr}(P B=n \mid D T=b, O S=b, P G=s, I C=i, F A=a, A B=<20)$ is "c2-1", and so on.

There were two concerns in knowledge elicitation. First, the authors knew that it was keenly important to achieve timely responses from the experts without frustrating them, and to maximize the likelihood that they would keep their commitment to participate over the duration of the 3 rounds. Therefore, high interactions between experts and the researchers were anticipated. Second, the experts should not consult with each other during the elicitation process, so low interactions between experts were desired. The personal interview has the highest degree of researcher-expert interaction and the lowest degree of expert-expert interference (Armstrong 1985). Therefore, personal interview was selected to elicit the conditional probabilities. A probability scale shown in Table 3 with both verbal and numerical expressions was used (Edwards, 1995). 
Table 2. Conditional probabilities elicited from airport experts in each round

\begin{tabular}{|c|c|c|c|c|c|}
\hline \multirow[t]{2}{*}{ No. } & \multirow{2}{*}{$\begin{array}{c}\text { Unfavorable } \\
\text { states }(j)\end{array}$} & \multirow[t]{2}{*}{ Probability of a scenario elicited } & \multicolumn{3}{|c|}{ Round } \\
\hline & & & 1 & 2 & 3 \\
\hline c1 & 0 & $\operatorname{Pr}(P B=n \mid D T=b, O S=b, P G=s, I C=i, F A=a, A B=>20)$ & $\mathrm{X}$ & & $\mathrm{X}$ \\
\hline c2 & 1 & $\operatorname{Pr}(P B=n \mid D T=b, O S=b, P G=s, I C=i, F A=a, A B=<20)$ & & $\mathrm{X}$ & $\mathrm{X}$ \\
\hline c3 & 1 & $\operatorname{Pr}(P B=n \mid D T=b, O S=g, P G=s, I C=i, F A=a, A B=>20)$ & & & $\mathrm{X}$ \\
\hline c4 & 1 & $\operatorname{Pr}(P B=n \mid D T=b, O S=b, P G=s, I C=i, F A=t, A B=>20)$ & & $x$ & $\mathrm{X}$ \\
\hline c5 & 1 & $\operatorname{Pr}(P B=n \mid D T=b, O S=b, P G=s, I C=d, F A=a, A B=>20)$ & & & $\mathrm{X}$ \\
\hline c6 & 1 & $\operatorname{Pr}(P B=n \mid D T=b, O S=b, P G=g, I C=i, F A=a, A B=>20)$ & & & $x$ \\
\hline c7 & 2 & $\operatorname{Pr}(P B=n \mid D T=b, O S=g, P G=s, I C=i, F A=a, A B=<20)$ & & & $X$ \\
\hline c8 & 2 & $\operatorname{Pr}(P B=n \mid D T=b, O S=g, P G=s, I C=i, F A=t, A B=>20)$ & $x$ & $x$ & $x$ \\
\hline c9 & 2 & $\operatorname{Pr}(P B=n \mid D T=b, O S=g, P G=s, I C=d, F A=a, A B=>20)$ & & $x$ & $x$ \\
\hline c10 & 2 & $\operatorname{Pr}(P B=n \mid D T=b, O S=b, P G=s, I C=d, F A=t, A B=>20)$ & & $\mathrm{X}$ & $X$ \\
\hline c11 & 2 & $\operatorname{Pr}(\mathrm{PB}=\mathrm{n} \mid \mathrm{DT}=\mathrm{b}, \mathrm{OS}=\mathrm{b}, \mathrm{PG}=\mathrm{s}, \mathrm{IC}=\mathrm{i}, \mathrm{FA}=\mathrm{t}, \mathrm{AB}=<20)$ & & & $x$ \\
\hline$c 12$ & 2 & $\operatorname{Pr}(\mathrm{PB}=\mathrm{n} \mid \mathrm{DT}=\mathrm{b}, \mathrm{OS}=\mathrm{b}, \mathrm{PG}=\mathrm{s}, \mathrm{IC}=\mathrm{d}, \mathrm{FA}=\mathrm{a}, \mathrm{AB}=<20)$ & & & $x$ \\
\hline c13 & 2 & $\operatorname{Pr}(P B=n \mid D T=b, O S=g, P G=g, I C=i, F A=a, A B=>20)$ & $X$ & & $\mathrm{X}$ \\
\hline$c 14$ & 2 & $\operatorname{Pr}(\mathrm{PB}=\mathrm{n} \mid \mathrm{DT}=\mathrm{b}, \mathrm{OS}=\mathrm{b}, \mathrm{PG}=\mathrm{g}, \mathrm{IC}=\mathrm{i}, \mathrm{FA}=\mathrm{t}, \mathrm{AB}=>20)$ & & $\mathrm{x}$ & $x$ \\
\hline c15 & 2 & $\operatorname{Pr}(P B=n \mid D T=b, O S=b, P G=g, I C=d, F A=a, A B=>20)$ & & $x$ & $x$ \\
\hline c16 & 2 & $\operatorname{Pr}(P B=n \mid D T=b, O S=b, P G=g, I C=i, F A=a, A B=<20)$ & & & $x$ \\
\hline c17 & 3 & $\operatorname{Pr}(\mathrm{PB}=\mathrm{n} \mid \mathrm{DT}=\mathrm{b}, \mathrm{OS}=\mathrm{g}, \mathrm{PG}=\mathrm{s}, \mathrm{IC}=\mathrm{i}, \mathrm{FA}=\mathrm{t}, \mathrm{AB}=<20)$ & & $\mathrm{X}$ & $\mathrm{X}$ \\
\hline c18 & 3 & $\operatorname{Pr}(P B=n \mid D T=b, O S=g, P G=s, I C=d, F A=a, A B=<20)$ & & & $x$ \\
\hline c19 & 3 & $\operatorname{Pr}(\mathrm{PB}=\mathrm{n} \mid \mathrm{DT}=\mathrm{b}, \mathrm{OS}=\mathrm{b}, \mathrm{PG}=\mathrm{s}, \mathrm{IC}=\mathrm{d}, \mathrm{FA}=\mathrm{t}, \mathrm{AB}=<20)$ & $\mathrm{X}$ & & $x$ \\
\hline c20 & 3 & $\operatorname{Pr}(P B=n \mid D T=b, O S=g, P G=s, I C=d, F A=t, A B=>20)$ & & & $\mathrm{x}$ \\
\hline$c 21$ & 3 & $\operatorname{Pr}(P B=n \mid D T=b, O S=g, P G=g, I C=i, F A=t, A B=>20)$ & & & $x$ \\
\hline $\mathrm{c} 22$ & 3 & $\operatorname{Pr}(P B=n \mid D T=b, O S=g, P G=g, I C=i, F A=a, A B=<20)$ & & & $x$ \\
\hline c23 & 3 & $\operatorname{Pr}(P B=n \mid D T=b, O S=b, P G=g, I C=d, F A=a, A B=<20)$ & $X$ & $\mathrm{X}$ & $X$ \\
\hline $\mathrm{c} 24$ & 3 & $\operatorname{Pr}(P B=n \mid D T=b, O S=g, P G=g, I C=d, F A=a, A B=>20)$ & & & $\mathrm{X}$ \\
\hline c25 & 3 & $\operatorname{Pr}(\mathrm{PB}=\mathrm{n} \mid \mathrm{DT}=\mathrm{b}, \mathrm{OS}=\mathrm{b}, \mathrm{PG}=\mathrm{g}, \mathrm{IC}=\mathrm{i}, \mathrm{FA}=\mathrm{t}, \mathrm{AB}=<20)$ & & $\mathrm{X}$ & $\mathrm{x}$ \\
\hline c26 & 3 & $\operatorname{Pr}(P B=n \mid D T=b, O S=b, P G=g, I C=d, F A=t, A B=>20)$ & & $x$ & $x$ \\
\hline c27 & 4 & $\operatorname{Pr}(P B=n \mid D T=b, O S=g, P G=s, I C=d, F A=t, A B=<20)$ & & $\mathrm{X}$ & $\mathrm{X}$ \\
\hline c28 & 4 & $\operatorname{Pr}(\mathrm{PB}=\mathrm{n} \mid \mathrm{DT}=\mathrm{b}, \mathrm{OS}=\mathrm{g}, \mathrm{PG}=\mathrm{g}, \mathrm{IC}=\mathrm{i}, \mathrm{FA}=\mathrm{t}, \mathrm{AB}=<20)$ & & & $x$ \\
\hline c29 & 4 & $\operatorname{Pr}(P B=n \mid D T=b, O S=g, P G=g, I C=d, F A=a, A B=<20)$ & & $\mathrm{X}$ & $\mathrm{X}$ \\
\hline c30 & 4 & $\operatorname{Pr}(P B=n \mid D T=b, O S=g, P G=g, I C=d, F A=t, A B=>20)$ & & 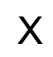 & $X$ \\
\hline c31 & 4 & $\operatorname{Pr}(P B=n \mid D T=b, O S=b, P G=g, I C=d, F A=t, A B=<20)$ & & & $X$ \\
\hline c32 & 5 & $\operatorname{Pr}(P B=n \mid D T=b, O S=g, P G=g, I C=d, F A=t, A B=<20)$ & $\mathrm{X}$ & & $\mathrm{X}$ \\
\hline
\end{tabular}

Table 3. Probability elicitation scale

\begin{tabular}{|l|l|l|l|l|l|l|l|l|l|l|}
\hline \multicolumn{2}{|l}{ Impossible Improbable } & \multicolumn{1}{c}{ Uncertain } & $50-50$ & \multicolumn{3}{c|}{ Somewhat expected } & \multicolumn{3}{c|}{ Probable Certain } \\
\hline $1 \%$ & $10 \%$ & $20 \%$ & $30 \%$ & $40 \%$ & $50 \%$ & $60 \%$ & $70 \%$ & $80 \%$ & $90 \%$ & $99 \%$ \\
\hline
\end{tabular}




\section{DATA EXAMINATION}

There are two underlying assumptions to this research. First, experts, by the very nature of being experts, have fairly consistent beliefs in their knowledge domain. Second, fractional or incomplete data can be representative of the whole. To check these assumptions, the data were examined in three ways: inter-consistency, intra-consistency, and frequency of cited probabilities. The experts' performance against these three measures is discussed at the end of this section.

\section{Inter-consistency}

The inter-consistency of an expert refers to the consistency of probabilities provided by the expert for the same scenario but in different rounds. Inter-consistency can be measured by the difference of probabilities between rounds. If the difference between rounds is $0 \%$, the interconsistency of the expert is perfect. The levels of inter-consistency shown in Table 4 were set somewhat arbitrarily.

Table 4. Consistency levels

\begin{tabular}{llcccc}
\hline Criteria & Measure & Very good & Good & Borderline & Poor \\
\hline Inter-consistency & Average difference & $<10 \%$ & $10-15 \%$ & $15-25 \%$ & $>25 \%$ \\
Intra-consistency & Abnormal pairs & $0-3$ & $4-6$ & $7-9$ & $>9$ \\
\hline
\end{tabular}

Experts were not informed of the consistency test, therefore they were not pressured to check their answers from the earlier rounds to conform with them. Probabilities elicited in rounds 1, 2 and 3 are shown in the Appendix. Table 5 shows the absolute differences between round 3 and round 1 for each expert, and Table 6 shows the absolute differences between round 3 and round 2 for each expert. The average absolute differences are shown in the last row of each table.

Table 5. Absolute differences between round 3 and round 1 for each expert

\begin{tabular}{lrrrrrrr}
\hline Probability & E1 & E2 & E3 & E4 & E5 & E6 & E7 \\
\hline c1-0 & 0 & 0 & 9 & 10 & 0 & 9 & 0 \\
c8-2 & 0 & 20 & 10 & 0 & 10 & 10 & 30 \\
c13-2 & 40 & 20 & 10 & 0 & 10 & 0 & 10 \\
c19-3 & 10 & 0 & 20 & 0 & 10 & 0 & 30 \\
c23-3 & 20 & 50 & 40 & 10 & 0 & 0 & 20 \\
c32-5 & 9 & 10 & 30 & 10 & 20 & 30 & 9 \\
No. $>25 \%$ & 1 & 1 & 2 & 0 & 0 & 1 & 2 \\
Average & 13.2 & 16.7 & 19.8 & 5.0 & 8.3 & 8.2 & 16.5 \\
\hline
\end{tabular}

Table 6. Absolute differences between round 3 and round 2 for each expert

\begin{tabular}{lrrrrrrr}
\hline Probability & E1 & E2 & E3 & E4 & E5 & E6 & E7 \\
\hline c2-1 & 0 & 0 & 20 & 0 & 10 & 9 & 0 \\
c4-1 & 0 & 0 & 10 & 0 & 0 & 0 & 0 \\
c8-2 & 20 & 0 & 10 & 0 & 10 & 10 & 0 \\
c9-2 & 10 & 0 & 40 & 0 & 0 & 10 & 20 \\
\hline
\end{tabular}




\begin{tabular}{lrrrrrrr}
\hline Probability & E1 & E2 & E3 & E4 & E5 & E6 & E7 \\
\hline c10-2 & 30 & 20 & 20 & 0 & 0 & 10 & 0 \\
c14-2 & 10 & 0 & 0 & 0 & 0 & 10 & 10 \\
c15-2 & 0 & 10 & 30 & 10 & 20 & 30 & 30 \\
c17-3 & 10 & 0 & 10 & 0 & 0 & 10 & 10 \\
c23-3 & 0 & 0 & 0 & 0 & 10 & 40 & 30 \\
c25-3 & 10 & 0 & 10 & 0 & 20 & 20 & 10 \\
c26-3 & 0 & 30 & 0 & 0 & 0 & 20 & 30 \\
c27-4 & 9 & 0 & 40 & 0 & 10 & 0 & 10 \\
c29-4 & 19 & 20 & 0 & 0 & 10 & 10 & 10 \\
c30-4 & 0 & 0 & 10 & 0 & 20 & 30 & 30 \\
No. >25\% & 1 & 1 & 3 & 0 & 0 & 3 & 4 \\
Average & 8.4 & 5.7 & 14.3 & 0.7 & 7.9 & 14.9 & 13.6 \\
\hline & & & & & & &
\end{tabular}

The differences for each expert are trivial for most scenarios (only 19 out of 140 are over $25 \%$ and all averages under $20 \%$ ), and there are no obvious trends between rounds. Although an expert may propose different probabilities for the same scenario, the ranges of differences are relatively narrow and typically do not change the preference to the scenario though the degree of intensity changes quantitatively. For example, E1 believed that $\operatorname{Pr}(P B=n \mid D T=b, O S=b$, $P G=s, I C=d, F A=t, A B=<20$ ) should be $20 \%$ in round 1 whereas $s /$ he changed the probability for the same scenario to $10 \%$ in round 3 . Although $\mathrm{E} 1$ gave different numbers in the two rounds, this expert believed that the probability of constructing a new passenger building in this scenario was rather low. The inter-consistency of the belief of an expert suggests that further research focusing on the mechanism of probability elicitation within an expert rather than across experts is needed.

It was decided to investigate if the number of questions in the survey affected the interconsistency for each expert. Figure 4 compares the average absolute difference between round 3 and round 1 and between round 3 and round 2 . As shown, the inter-consistency varies for each expert, with 7 under $10 \%$, 4 between $10 \%$ and $15 \%$, 3 between $15 \%$ and $20 \%$.

Considering both rounds, E4 shows the best inter-consistency, E3 shows the least interconsistency, but all are under $20 \%$. Therefore, all experts can be considered inter-consistent if a criterion of $25 \%$ is adopted.

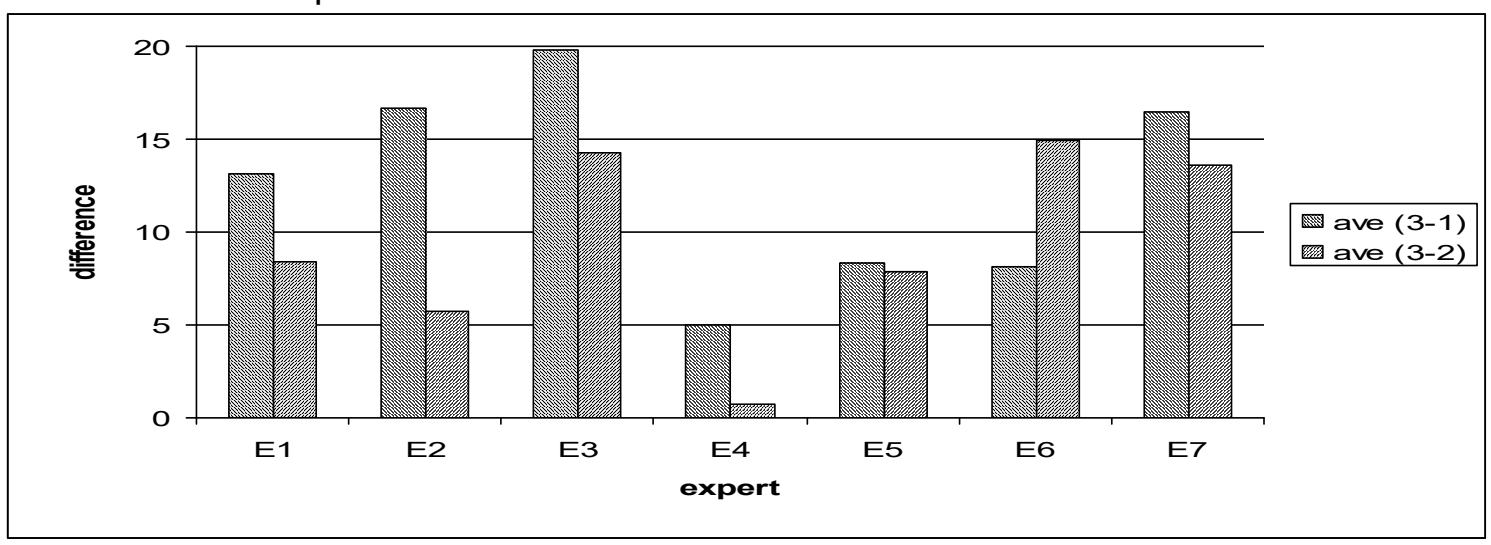

Figure 4. Averages of the absolute differences by expert 
There is no obvious advantage for either round 1 or round 2, since the average absolute differences fluctuate within a narrow range for every expert. The average difference between round 1 and round 3 of the experts is $12.5 \%$, and the average difference between rounds 2 and 3 is $9.4 \%$. Given that the difference is trivial and there is no distinct benefit in extrapolating the whole domain structure from the intermediate set of 14 scenarios, the concise set of 6 scenarios is preferred.

\section{Intra-consistency}

The intra-consistency of an expert refers to how rational the probabilities are for different scenarios in the same round. It can be measured by the difference of probabilities for a pair of comparable scenarios, which is defined as two scenarios that have identical unfavorable states but one of them has an extra unfavorable state. Theoretically, the scenario with the extra unfavorable state should have a probability less than or equal to the former. For example, because c20-3 and c27-4 share same unfavorable states (OS=g, IC=d, FA=t), but c27-4 has one more unfavorable state $(A B=<20)$, they are a pair of comparable scenarios. If the probability of $\mathrm{c} 27-4$ is less than or equal to that of $\mathrm{c} 20-3$, then this pair is a normal pair; however, if the probability of $c 27-4$ is higher than that of c20-3, then they are called an abnormal pair. If no abnormal pairs are found within a round from an expert, the intra-consistency of the expert is "very good". With the increase of abnormal pairs from an expert, the intra-consistency of the expert worsens. All 80 comparable scenario pairs were tested. The levels of intra-consistency were set arbitrarily (Table 4). The number of abnormal pairs detected in the responses from each expert are listed in Table 7. Only expert E2 had poor intra-consistency, i.e., >9 abnormal pairs.

Table 7. Intra-consistency of each expert

\begin{tabular}{lccccccc}
\hline Expert & E1 & E2 & E3 & E4 & E5 & E6 & E7 \\
\hline No. of abnormal pairs & 2 & 16 & 4 & 0 & 9 & 1 & 1 \\
\hline
\end{tabular}

\section{Frequency of cited probabilities}

In round 3 , the experts responded to all 32 scenarios, showing different patterns in the probability distribution. For example, E1 gave the lowest probability in most scenarios; E2 showed some jumps in probabilities when certain factors changed from favorable to unfavorable states. Figure 5 shows how often each expert cited a probabilistic value, indicating the general pessimism or optimism of an expert.

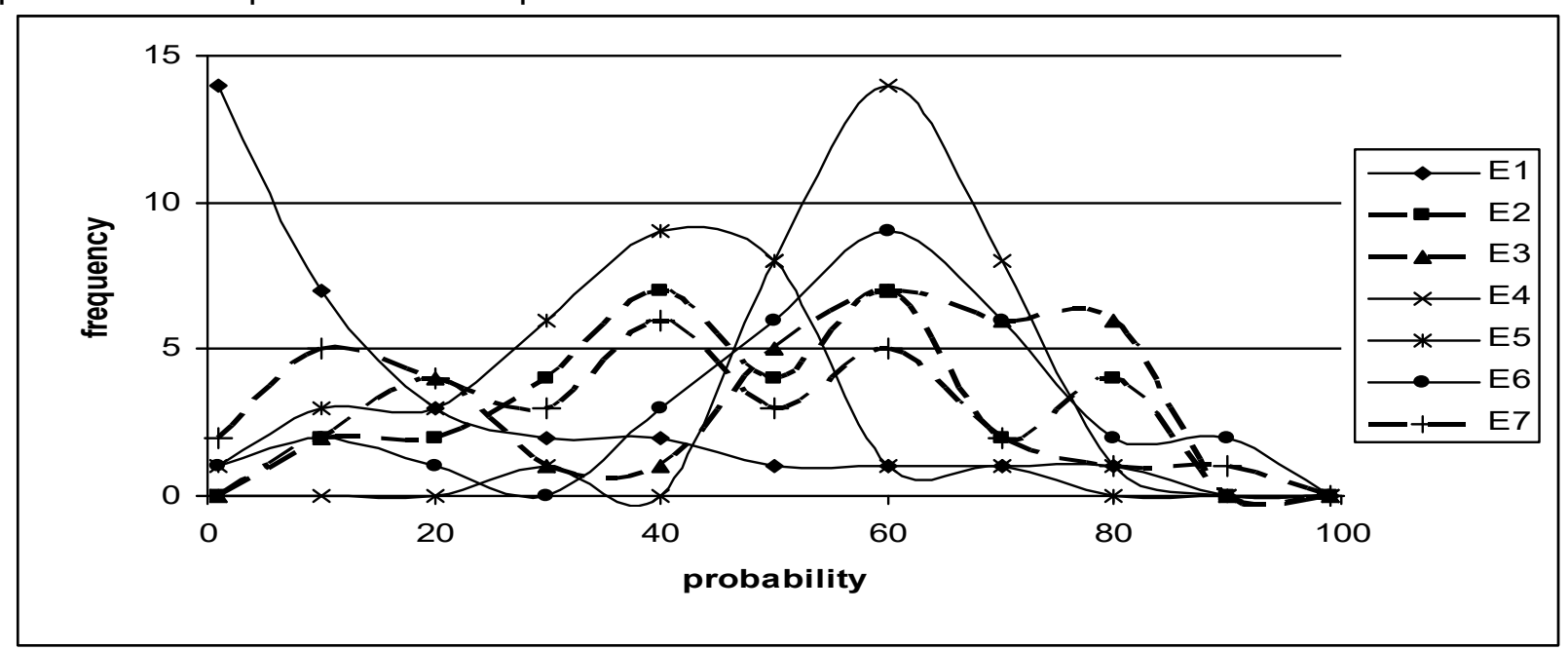

Figure 5. The frequency of probabilities in round 3 cited by each expert 
As shown in Figure 5, E1 has a high concentration of probabilities between $1 \%$ and $20 \%$, which shows an adverse attitude toward building a new passenger building. E4, E5 and E6 also have one peak (but centered on the distribution) with the frequencies rapidly reducing in both directions, though the most frequently cited probability value changes. This pattern implies that $\mathrm{E} 1, \mathrm{E} 4, \mathrm{E} 5$ and E6 have a tendency to anchor at certain options. Compared to the others, E2, E3 and E7 (shown with heavy dashed lines) have a relatively uniform distribution of probabilities along the whole range although the frequencies fluctuate. This indicates that E2, E3 and E7 take the influence of favorable and unfavorable factors on their beliefs of constructing a new passenger building somewhat linearly.

As expected, probabilities generally decrease as the number of unfavorable states increases, as shown in Figure 6, though the shape of the curve changes depending on the expert. The uniform distributions of E2, E3, and E7 show a linear shape compared to one peak distributions from E1, E4, E5, and E6.

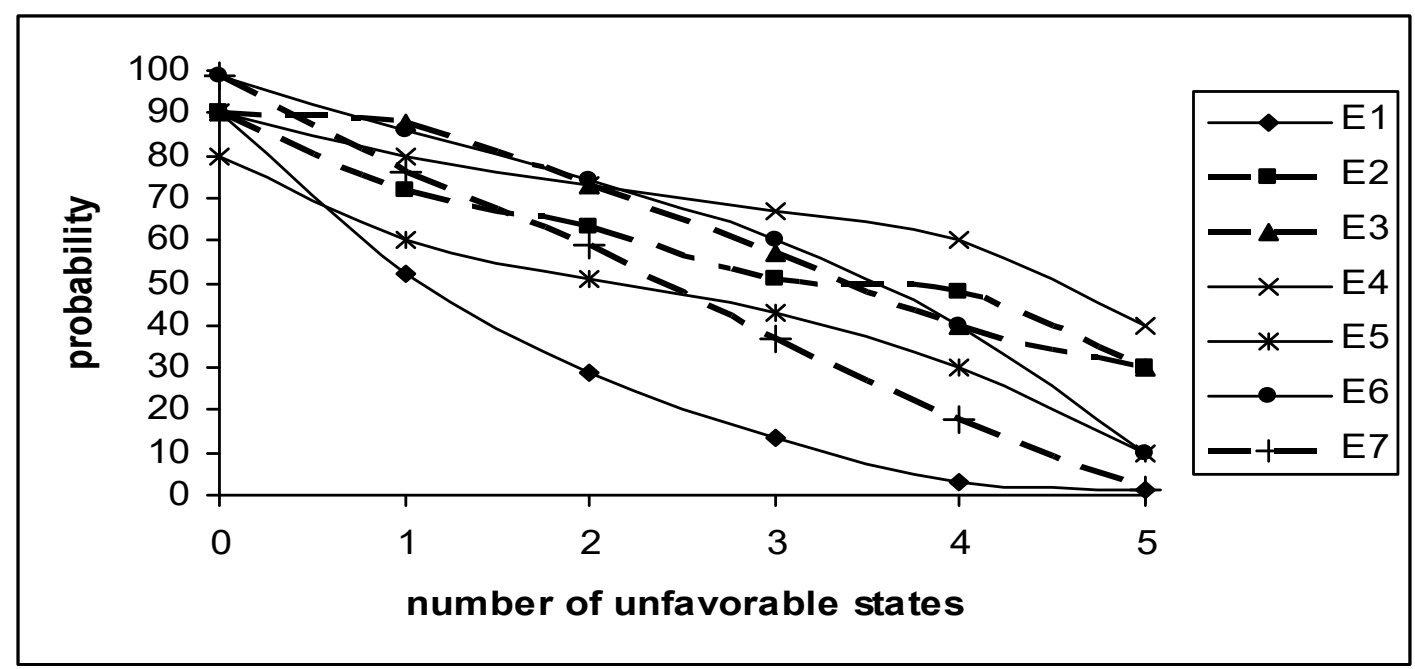

Figure 6. The average probabilities with unfavorable states for each expert

\section{Summary of data examination}

The three examination criteria are summarized in Table 8. The first assumption that each expert has fairly consistent beliefs in their knowledge domain is supported by their inter-consistency and distribution pattern. The second that fractional or incomplete data can be representative of the whole is supported by the intra-consistency and distribution patterns.

Table 8. The characteristics of probabilities elicited from experts

\begin{tabular}{llll}
\hline Expert & Distribution pattern & Inter-consistency & Intra-consistency \\
\hline E1 & One peak & Good & Very good \\
E2 & Uniform & Good & Poor \\
E3 & Uniform & Borderline & Good \\
E4 & One peak & Very good & Very good \\
E5 & One peak & Very good & Borderline \\
E6 & One peak & Good & Very good \\
E7 & Uniform & Borderline & Very good \\
\hline
\end{tabular}




\section{ESTIMATING COMPLETE CONDITIONAL PROBABILITY TABLES}

As observed, the probability value decreases when the number of unfavorable states of influencing factors increases in an approximately linear or curvilinear way. Therefore, methods of predicting unknown values by interpolating elicited knowledge can be developed. The benefit will be evident if the method of prediction can be made as simple as possible while still being effective. Three methods for establishing the missing probabilities of round 1 were tested: 1) setting all missing values to $50 \%, 2$ ) linear interpolation between the maximum and minimum values, and 3) piecewise representation. As observed earlier, the concise set of scenarios in round 1 provided reasonably similar inter-consistency as the intermediate efforts of round 2. Therefore, round 1 is used in the hypothesis testing undertaken in this section.

\section{$50 \%$ representation (Method 1)}

Where the probability may not be sensitive to the states of influencing factors, it might be possible to use $50 \%$ to represent those situations when experts' opinions are not available. In Method 1, the probability values absent in round 1 are assigned $50 \%$ so that a whole spectrum for the domain can be predicted. The differences between the predicted values in Method 1 and the feedback in round 3 can be seen in Table 9.

Table 9. Predictions of Method 1 compared to Round 3

\begin{tabular}{lrrrrrrr}
\hline Item & E1 & E2 & E3 & E4 & E5 & E6 & E7 \\
\hline Average difference & 21.7 & -6.6 & -12.2 & -16.6 & 3.8 & -12.2 & 2.2 \\
Standard deviation & 22.7 & 15.6 & 19.6 & 10.7 & 12.9 & 18.9 & 20.4 \\
Average absolute difference & 27.9 & 14.7 & 22.2 & 17.5 & 10.9 & 18.7 & 18.4 \\
Maximum error & 49.0 & 50.0 & 40.0 & 30.0 & 30.0 & 49.0 & 40.0 \\
No. of diff. $>25 \%$ & 20 & 5 & 12 & 8 & 3 & 12 & 11 \\
\hline
\end{tabular}

The difference between Method 1 predictions and round 3 is more than $25 \%$ in 71 out of 210 (30 scenarios predicted $\times 7$ experts) cases. Therefore, it is not appropriate to assume an unknown probability as $50 \%$ when data are absent.

\section{Linear interpolation (Method 2)}

Since experts use the scenario with no unfavorable states as the benchmark and then reduce the probability for scenarios with increasing unfavorable states, a method of simple linear interpolation can be explored. For each expert, equations ( 2 ) and ( 3 ) were used to compute the probability value of an unknown scenario based on the maximum and minimum values elicited from the expert.

First, the interval for each unfavorable state was determined using Eq. ( 2 ), where $j$ is the number of unfavorable states. In this case, $j(\min )=0, j(\max )=5$. Then, the unknown probability values for each number of unfavorable states was predicted using Eq. ( 3 ).

$$
\begin{gathered}
\text { interval }=\frac{\operatorname{Pr}(j(\min ))-\operatorname{Pr}(j(\max ))}{j(\max )} \\
\operatorname{Pr}(j=1 \ldots(j(\max )-1))_{\text {predicted }}=\operatorname{Pr}(j(\min ))-j * \text { interval }
\end{gathered}
$$

In Table 10 are listed for each expert: the interval calculated by Eq. ( 2 ), maximum and minimum probability values, and the predicted probabilities $\operatorname{Pr}(j)$ based on Eq. ( 3 ). Also shown 
is the average and standard deviation of the difference between the values predicted by Method 2 and the actual feedback in round 3.

Table 10. Predictions of Method 2 compared to Round 3

\begin{tabular}{lrrrrrrrl}
\hline Item & E1 & E2 & E3 & E4 & E5 & E6 & E7 & Notes \\
\hline interval & 17.8 & 14 & 14 & 10 & 14 & 17.8 & 19.6 & Eq. ( 2 ) \\
$\operatorname{Pr}(0)$ & 90 & 90 & 90 & 90 & 80 & 99 & 99 & given \\
$\operatorname{Pr}(1)$ & 72 & 76 & 76 & 80 & 66 & 81 & 79 Eq. (3) \\
$\operatorname{Pr}(2)$ & 54 & 62 & 62 & 70 & 52 & 63 & 60 & Eq. (3) \\
$\operatorname{Pr}(3)$ & 37 & 48 & 48 & 60 & 38 & 46 & 40 & Eq. (3) \\
$\operatorname{Pr}(4)$ & 19 & 34 & 34 & 50 & 24 & 28 & 21 & Eq. (3) \\
$\operatorname{Pr}(5)$ & 1 & 20 & 20 & 40 & 10 & 10 & 1 & given \\
Average diff'ce & 20.9 & -3.1 & -9.4 & -4.7 & -1.3 & -10.4 & 2.2 & \\
Standard deviation & 14.8 & 17.2 & 14.5 & 5.1 & 10.3 & 11.7 & 9.4 & \\
Average absolute diff'ce & 22.0 & 14.6 & 15.4 & 4.7 & 8.3 & 13.4 & 7.3 & \\
Max. absolute error & 44.4 & 36.0 & 32.0 & 10.0 & 26.0 & 32.2 & 20.2 & \\
No. of diff.>25\% & 15 & 5 & 4 & 0 & 1 & 1 & 0 & \\
\hline
\end{tabular}

Only 26 out of 210 predicted scenarios exceed the threshold of $25 \%$; however more than half the cases occurred with E1. Therefore, Method 2 may not work well if the probability distribution is non-linear, as was evidenced with E1. Compared to Method 1, Method 2 leads to better prediction for every expert.

\section{Piecewise representation (Method 3)}

A piecewise representation was developed in Method 3, where fractional data were elicited from experts for each set of scenarios with the same number of unfavorable states. If no data were elicited for a specific number of unfavorable states, the missing data were interpolated between the nearest two data using Eqs.( 2 ) and ( 3 ), but substituting the nearest data values for the maximum and minimum values. Generally, the process is:

1. If one probabilistic value is available from the expert for scenarios with the same number of unfavorable states, use that value for all scenarios with the same number of unfavorable states.

2. If two or more probabilistic values are available from the expert for scenarios with the same number of unfavorable states, use the average of the values for all scenarios with the same number of unfavorable states.

3. For the scenarios with the same number of unfavorable states where no probabilistic values from the expert are available, use linearly-interpolated values from the two adjacent numbers of unfavorable states to predict the probabilities, i.e.,

$$
\operatorname{Pr}(j)=(\operatorname{Pr}(j-1)+\operatorname{Pr}(j+1)) / 2
$$

where $\operatorname{Pr}(j)$ is the probability for $j$ unfavorable states.

Based on these rules, $\operatorname{Pr}(j=0$ to 5$)$ were determined for each expert, as shown in Table 11 . Only 12 out of 224 differences between the predicted values from Method 3 and the feedback in round 3 exceed the threshold of $25 \%$. No evident trend can be observed in terms of systemic errors, i.e., no consistent over-estimation or under-estimation in the prediction can be identified. 
Table 11. Predictions of Method 3 compared to Round 3

\begin{tabular}{lrrrrrrrl}
\hline Item & E1 & E2 & E3 & E4 & E5 & E6 & E7 & Notes \\
\hline $\operatorname{Pr}(0)$ & 90 & 90 & 90 & 90 & 80 & 99 & 99 & given \\
$\operatorname{Pr}(1)$ & 65 & 78 & 90 & 80 & 73 & 87 & 72 & Eq. (4) \\
$\operatorname{Pr}(2)$ & 40 & 65 & 90 & 70 & 65 & 75 & 45 & average \\
$\operatorname{Pr}(3)$ & 20 & 55 & 75 & 60 & 45 & 70 & 40 & average \\
$\operatorname{Pr}(4)$ & 11 & 38 & 48 & 50 & 28 & 40 & 21 & Eq. (4) \\
$\operatorname{Pr}(5)$ & 1 & 20 & 20 & 40 & 10 & 10 & 1 & given \\
Average error & 8.9 & 0.9 & 12.2 & -4.7 & 6.7 & 3.6 & -3.6 & \\
St'd deviation & 13.8 & 17.2 & 16.2 & 5.1 & 11.5 & 11.8 & 12.0 & \\
Ave. abs. Diff. & 13.2 & 14.4 & 13.7 & 4.7 & 10.3 & 8.8 & 9.4 & \\
Max. error & 35.0 & 35.0 & 60.0 & 10.0 & 35.0 & 40.0 & 25.0 & \\
No. of diff.>25\% & 3 & 3 & 4 & 0 & 1 & 1 & 0 & \\
\hline
\end{tabular}

\section{Comparison of Methods 1,2 and 3}

The averages and maximums of the absolute difference between the values from Methods 1, 2 $\& 3$ and round 3 over 32 scenarios for each expert are repeated in Table 12 for comparative purposes. The minimum difference was zero in all cases.

Table 12. Comparisons of Methods 1, 2 and 3

\begin{tabular}{llrrrrrrr}
\hline & & E1 & E2 & E3 & E4 & E5 & E6 & E7 \\
\hline Method 1 & average prediction error & 27.9 & 14.7 & 22.2 & 17.5 & 10.9 & 18.7 & 18.4 \\
& maximum error & 49.0 & 50.0 & 40.0 & 30.0 & 30.0 & 49.0 & 40.0 \\
& number of differences $>$ 25\% & 20 & 5 & 12 & 8 & 3 & 12 & 11 \\
\hline Method 2 & average prediction error & 22.0 & 14.6 & 15.4 & 4.7 & 8.3 & 13.4 & 7.3 \\
& maximum error & 44.4 & 36.0 & 32.0 & 10.0 & 26.0 & 32.2 & 20.2 \\
& number of differences > 25\% & 15 & 5 & 4 & 0 & 1 & 1 & 0 \\
\hline Method 3 & average prediction error & 13.2 & 14.4 & 13.7 & 4.7 & 10.3 & 8.8 & 9.4 \\
& maximum error & 35.0 & 35.0 & 60.0 & 10.0 & 35.0 & 40.0 & 25.0 \\
& number of differences $>$ 25\% & 3 & 3 & 4 & 0 & 1 & 1 & 0 \\
\hline
\end{tabular}

Method 3 has the best predictive performance among the methods, particularly for $\mathrm{E} 1$, as shown in Figure 7, because E1 deviated most from a linear relationship. It can be generalized, therefore, that piecewise representation adjusts the slope of each segment accordingly, which enhances the flexibility of the method and reduces errors. For the remaining experts, Method 2 and Method 3 showed similar predictive accuracy. E4 has identical accuracy in the predictions because it shows a good linear relationship between the probability values and the number of unfavorable states of the influencing factors; inter- and intra-consistency for E4 was also very high. This indicates that Method 2 and Method 3 produce similar results when the expert is consistent and shows a linear relationship between the probability and the number of unfavorable states of influencing factors. Considering the improvement of Method 3 against Method 2 for E1, Method 3 has a potential to predict unknown probability distributions more accurately. Therefore, piecewise representation method is preferred for predicting the probability of unknown scenarios based on the knowledge elicited from a domain expert. 


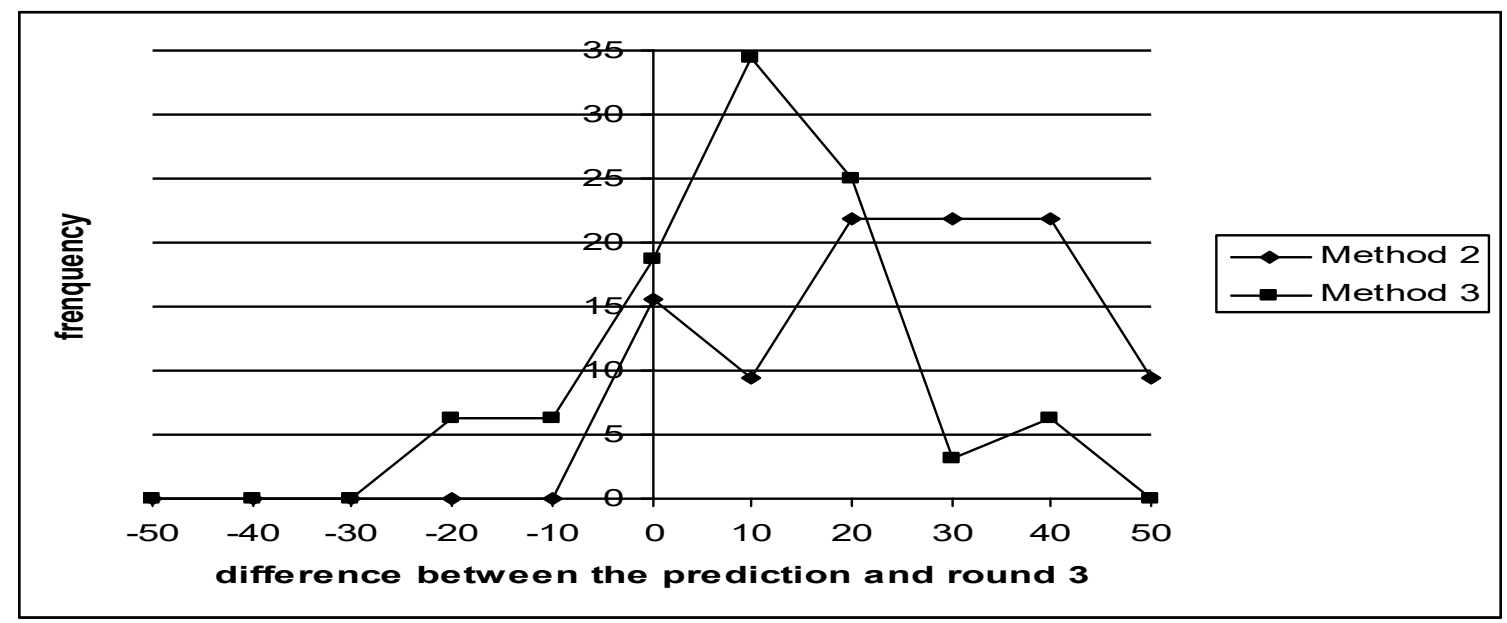

Figure 7. Distribution of the differences for E1

\section{APPLYING PIECEWISE REPRESENTATION IN A GENERAL CASE}

Suppose a BBN has independent parent nodes $\mathrm{P} 1, \mathrm{P} 2, \ldots, \mathrm{Pk}$ and one child node $\mathrm{C}$ as shown in Error! Reference source not found.. Since the number of states for a node does not affect the nature of a BBN, all nodes are assumed binary (true and false for child node; favorable state and unfavorable state for the parent nodes). The number of probabilities required to quantify the child can be determined by Eq. ( 1 ). To demonstrate the general case, a Naïve Bayes net was set up with $k=9$, i.e. $N P=512$. Because it is very difficult for a domain expert to provide so many probabilities, the probabilities required to complete the CPT of the Naïve Bayes network were generated for demonstration purposes using Monte Carlo simulation.

\section{Determining the parameters in Monte Carlo simulation}

To perform the Monte Carlo simulations, three parameters are needed: 1) the number of probabilities for each number of unfavorable states; 2) the probability distribution, and, 3) the distribution bounds. The characteristics of the probability distribution in Monte Carlo simulations were based on the responses from the airport development experts.

The total number of probabilities for each combination of unfavorable states can be determined by Eq. $(5)$, where $j$ is the number of unfavorable states in the parent nodes, and $k$ is the number of parent nodes.

$$
N_{j}=\frac{k !}{j !(k-j) !}
$$

Because no trend in the probability distribution of experts was evident, a uniform distribution was assigned. The difference between the maximum and the minimum for scenarios under same number of unfavorable states averaged about $30 \%$ except at the extremes (i.e. the number of unfavorable states is all or none) where the difference was zero. Figure 8 shows the average of the probabilities from 7 experts against the number of unfavorable states. The curve indicates a very good linear relationship with a correlation coefficient of 0.999 . For comparison, the curve elicited from E1 is also plotted. The deviation of the curve from a linear relationship is noticeable. 


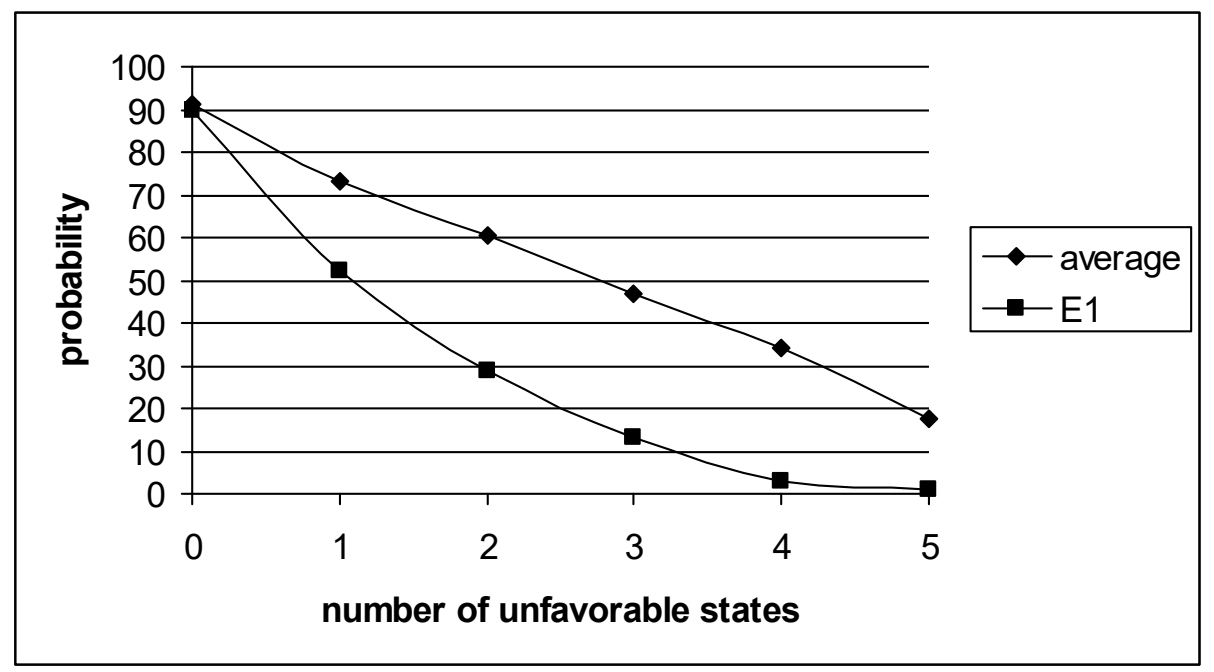

Figure 8. Probability vs. number of unfavorable states

The parameters used for the Monte Carlo simulations were:

- Uniform probability distributions;

- The mean of each distribution decreased evenly with the increase in unfavorable states;

- The width of each uniform distribution was $30 \%$.

\section{Comparing Method 2, Method 3 and Monte Carlo simulation}

Five simulations were carried out to model five domain experts. For each domain expert, linear interpolation (Method 2) and piecewise representation (Method 3) were tested against the data from the simulation. Three probabilities for each number of unfavorable states were generated and averaged to represent the remaining probabilities under each number of unfavorable states. This represents the situation in which three randomly selected scenarios under each number of unfavorable states are elicited from a domain expert.

Both Method 2 and Method 3 perform well in predicting unknown probabilities. The differences between the predictions and the simulations are concentrated between $-10 \%$ and $10 \%$.

In this research, the correlation between probability values and the number of unfavorable states drawn from airport experts showed a strong linear relationship. Therefore, both linear interpolation and piecewise representation attained good predictions. If an elicitation is carried out in a domain where the relationship between probability values and the number of unfavorable states is not linear (as in the case of expert E1), the two methods may show different predictions. Monte Carlo simulation was carried out based on the responses from E1. Method 3 provided better predictions for E1 than Method 2 did, which demonstrates that piecewise representation is more flexible and outperforms linear interpolation. Therefore, piecewise representation is recommended for a general elicitation.

\section{The procedure of piecewise representation}

For the airport case, the probabilities of scenarios with 1 and 4 unfavorable states in the piecewise representation were calculated by interpolation using the adjacent probabilities. To explore the effect of a partial interpolation in the method of the piecewise representation, a comparison was made using Monte Carlo simulations on a Naïve Bayes net with 9 parent nodes. Five simulations were performed to represent 5 domain experts. Two groups of predictions were compared with the simulations. In group 1, the probabilities of scenarios with 1 
unfavorable state and 8 favorable states were the average of the probabilities from the simulation as normal. In group 2, the probabilities of scenarios with 1 unfavorable state and 8 favorable states were not generated by Monte Carlo simulation. Instead, they were interpolated from probabilities of 0 and 2 unfavorable states and 7 and 9 unfavorable states respectively. Compared to the piecewise representation, a partial linear interpolation on 1 and 8 unfavorable states shows less accuracy. Out of 90 scenarios ( 9 scenarios $X 5$ simulations $X 2$ groups) predicted, a partial linear interpolation shows 25 scenarios without error. However, the piecewise representation shows 33 scenarios without error. The effort of elicitation saved in partial linear interpolation deteriorated the quality of the probability prediction. Therefore, to improve the accuracy of the prediction, it is recommended that the piecewise representation rather than partial linear interpolation be used in eliciting fractional data for the BBN construction.

In piecewise representation, a greater number of probability samples under each of the unfavorable states brings higher accuracy in the prediction, as observed in the inter-consistency results between round 1 and round 2 . However, it is also more costly in terms of the effort required for probability elicitation (6 versus 14 probabilities respectively). Therefore, a costeffective number of sampling probabilities under each number of unfavorable states may benefit the knowledge elicitation process. The differences between predictions from piecewise representation and Monte Carlo simulations when the number of samples varies from 1 to 6 under each number of unfavorable states were examined. Table 13 shows the average of the absolute difference between piecewise representation and Monte Carlo simulation under a different number of samples. It is seen that the accuracy of predictions improves noticeably when the number of samples increases from 1 to 2 , but the gain is insignificant when the number of samples increases from 2 to 6 . Therefore, the authors recommend that 2 samples under each number of unfavorable states be used.

Table 13. The absolute difference under different number of samples

\begin{tabular}{lrrrrrr}
\hline Number of samples & 1 & 2 & 3 & 4 & 5 & 6 \\
\hline Average of the absolute difference & 10 & 7.9 & 7.9 & 7.5 & 7.4 & 7.5 \\
\hline
\end{tabular}

The predictive procedure with piecewise representation can be applied to knowledge elicitation of Bayesian belief networks with one child node and $k$ non-dominant parent nodes. This method reduced the number of probabilities required for elicitation to define the CPT from $2^{k}$ as shown in Eq. ( 1 ) to $2 k$ (assuming binary nodes). The steps for generalized piecewise representation are:

1. Elicit the probability of the scenario with 0 unfavorable states and the probability of the scenario with $k$ unfavorable states to anchor the extremes.

2. Elicit the probabilities of two scenarios with $j$ unfavorable states, $j=1, \ldots,(k-1)$. The choice of exactly which two scenarios for each $j$ to present to the experts in practice can be arbitrary; however, if a certain scenario is more common or sensitive in decision making, then it should be included. As discussed earlier, the savings gained by omitting certain $j$ from the elicitation process (and using partial interpolation to evaluate the missing probabilities) increases error; therefore, the authors recommend that two probabilities be elicited for every $j$.

3. Use the average of the two scenario probabilities with $j$ unfavorable states, $j=1, \ldots,(k-1)$, to represent the probabilities of other scenarios with $j$ unfavorable states, $j=1, \ldots,(k-1)$. If the difference between the two scenarios under the same number of unfavorable states is greater than the $25 \%$ threshold, then the existence of an important factor should be investigated, as discussed in the next section. 


\section{Piecewise representation with an important factor}

The method of piecewise representation can be used to estimate conditional probability tables in BBN without the existence of any dominant factor. As discussed earlier, when a dominant factor exists, the other factors do not affect the outcome. Therefore, the elicitation only needs to be conducted for scenarios when the dominant factor is off. In actual projects, it is possible that there exists one factor that is more influential than other factors but it does not dominate the others completely. This factor that balances between the dominant and non-dominant factors will be referred to as an "important factor".

When an important factor is identified in the process of knowledge elicitation, special attention needs to be paid to that factor. Usually a domain expert does not feel comfortable to give a ratio between an important factor and a non-dominant factor although s/he can single out the important factor qualitatively. To address the issue, the analyst can categorize all scenarios into two groups: one with the important factor on, and one with the important factor off. Domain experts can provide probabilities for both groups. For each group, the method of piecewise representation can be applied to estimate all scenarios in the group. The scenarios in both groups can be merged into one complete conditional probability table for the domain.

Note that each time an important factor is identified and addressed in this manner, the required number of probabilities from the experts approximately doubles and the efficiency of the piecewise representation method reduces. However, compared to 512 probabilities for the complete CPT of a Naïve Bayes net with 9 parent nodes, the savings gained by eliciting only 34 (number of parent nodes *2 -2 ) rather than 18 probabilities from the expert is still obvious.

In the practical elicitation of probabilities with an important factor, it is advisable to examine the probabilities after a complete CPT has been developed. The influence of an important factor can be estimated through comparisons among probabilities in the complete CPT. The estimation of a factor can be presented to domain experts for confirmation. If domain experts concur with the estimation, the elicited and estimated probabilities can be entered into a BBN. Otherwise, a new round of elicitation should be carried out. The process should be iterated until the estimation of an important factor meets the expectation of domain experts.

\section{The application of piecewise representation to general BBN}

A Naïve Bayes net is a basic type of BBN. When a complex BBN is explored, it usually contains Naïve Bayes nets in its local structure. Therefore, the achievement of this research based on Naïve Bayes networks can be applied to estimate conditional probabilities for a local structure in the complex BBN. No matter how complex a BBN is, when domain experts provide conditional probabilities for a node, they always need to look at the influence of its parent nodes in isolation of the rest of the network. Hence, the node and its parent nodes form a Naïve Bayes net, and domain experts deal with this local structure similarly as a separate Naïve Bayes. As a demonstration, a Tree Augmented Naïve Bayes is compared with a Naïve Bayes in Figure 9.

Conditional probabilities are associated with the structure of a BBN. $X$ and $Y$ will have different influences on $Z$ when they are instantiated in $(a)$ and $(b)$ in Figure 9, so $P(Z)$ has different meanings in (a) and (b). However, when the local conditional probabilities are examined, $P(Z \mid X=1, Y=1), P(Z \mid X=1, Y=0), P(Z \mid X=0, Y=1)$, and $P(Z \mid X=0, Y=0)$ are identical in both the Naïve Bayes and the Tree Augmented Naïve Bayes networks. In the view of knowledge elicitation, as long as $P(Z \mid X=1, Y=1), P(Z \mid X=1, Y=0), P(Z \mid X=0, Y=1)$, and $P(Z \mid X=0, Y=0)$ are sought, domain experts will only need to examine the local structure to provide conditional probabilities between a child node and its parent nodes. Therefore, the local conditional probability tables will not change with the global structure, and can be treated as Naïve Bayes. 
The method of piecewise representation can be used in the estimation of local conditional probabilities in complex BBN. The authors recommend that sensitivity analysis be performed on the network to identify nodes to which the anticipated output is particularly sensitive. If piecewise representation was used on those nodes, developers might consider additional efforts in the verification process.

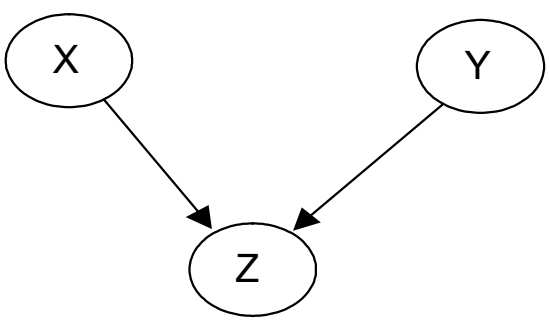

(a) Naïve Bayes

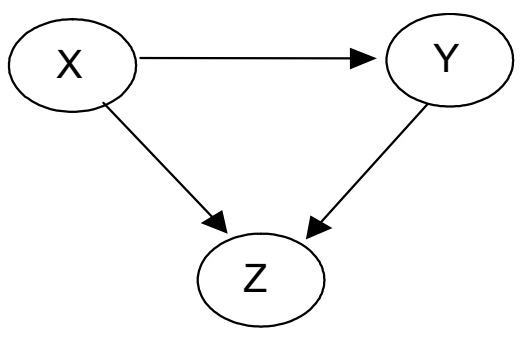

(b) Tree Augmented Naïve Bayes

Figure 9. Comparison of different BBN

\section{CONCLUSION}

A domain of airport development was used to establish a BBN for decision making. Based on the BBN, the techniques of using fractional data to develop complete conditional probability tables were proposed and examined. The inter-consistency between rounds of knowledge elicitation was quite high. This provided the opportunity to compare the results from the different rounds. The intra-consistency with respect to the number of abnormal pairs of scenarios depended upon the domain experts. Most domain experts showed fairly good intra-consistency.

Three methods of estimating a complete conditional probability table based on available fractional data from a domain expert were proposed and tested. The results showed that the linear interpolation method and the piecewise representation method provided good predictions in a linear domain. For a general domain, the piecewise representation method showed flexibility and outperformed the other methods. Considering the benefits of flexibility in general knowledge elicitation for BBN construction, the piecewise representation method is recommended for probability elicitation where the number of probabilities required for a single node is onerous. The piecewise representation method can be used to estimate the CPT of a node in general BBN.

The achievement of the research provides a solution to overcome a major barrier in using domain experts to supply a huge and intractable number of probabilities for BBN in engineering decision making. By using piecewise representation, the number of probabilities to be elicited for a binary child node with $k$ binary parent nodes is now $2 k$ rather than $2^{k}$.

\section{ACKNOWLEDGEMENT}

The authors gratefully acknowledge the financial support of IOR Grant \#216754-98 from the Greater Toronto Airports Authority and NSERC. Further, the authors sincerely thank MGP Project Managers and GTAA for their support and participation in this research. 


\section{REFERENCES}

Armstrong, J. S. (1985). Long-range forecasting, from crystal ball to computer, $2^{\text {nd }}$ ed., Wileyinterscience, John Wiley \& Sons, Inc., p112.

Di Bacco, M., D’Amore, G. \& Scalfari, F. (2004). Applied Bayesian Statistical Studies in Biology and Medicine. Kluwer Academic Publishers, Boston.

Druzdzel, M. J. \& Van der Gaag, L. C. (2000). Building probabilistic networks: "Where do the numbers come from?" Guest Editors' introduction, IEEE Transactions on Knowledge and Data Engineering, 12(4), 481-486.

Edwards, L. (1995). Practical risk management in the construction industry, Thomas Telford Publications. p.83.

Heckerman, D. (1995). A Tutorial on Learning with Bayesian Networks, Technical Report MSRTR-95-06, Microsoft Research.

Heckerman, D, Wellman, M. \& Mamdani, A. (Eds.) (1995). Communications of the ACM, 38(3).

Husmeier, D., Dybowski, R. \& Roberts, S. (Eds.) (2005). Probabilistic Modeling in Bioinformatics and Medical Informatics, Springer-Verlag, London.

McCabe, B., AbouRizk, S. M. and Goebel, R. (1998). Belief networks for construction performance diagnostics. J. Computing in Civil Engrg., ASCE, 12(2), 93-100.

Nasir, D., McCabe, B. and Hartono, L. (2003), Evaluating risk in construction-schedule model (ERIC-S): construction schedule risk model, J. Constr. Eng. Manage., ASCE, 129(5), 518-527.

O'Hagan, A. (1998). Eliciting expert beliefs in substantial practical applications, The Statistician, 47(1), 21-35.

Pearl, J. (1988). Probabilistic Reasoning in Intelligent Systems: networks of plausible inference, Morgan Kaufmann.

Sarkar, S. \& Murthy, I. (1996). Constructing efficient belief network structures with expert provided information, IEEE Transactions on Knowledge and Data Engineering, 8(1), 134-143.

Stutz, J., Taylor ,W. \& Cheeseman, P. (1998). AutoClass C - General Information, NASA, Ames Research Center.

Tang, Z. \& McCabe, B., (2002). Improving Risk Modeling Using Belief Networks, $30^{\text {th }}$ Annual Conference of the Canadian Society for Civil Engineering, Montréal, Québec, Canada, June $5-8,2002$.

Van der Gaag, L. C., Renooij, S, Witteman, C. L.M., Aleman, B. M. P. \& Taal, B. G. (1999). How to elicit many probabilities, Proceedings of the Fifteenth Conference on Uncertainty in Artificial Intelligence, 647-654.

Appendix A. Probabilities elicited from experts in round 1

\begin{tabular}{lllllllll}
\hline Scenario & $\#$ & E1 & E2 & E3 & E4 & E5 & E6 & E7 \\
\hline c1-0 & 0 & 90 & 90 & $\mathbf{9 9}$ & $\underline{80}$ & $\underline{80}$ & 90 & $\mathbf{9 9}$ \\
c8-2 & 2 & $\underline{30}$ & 70 & $\mathbf{9 0}$ & $\mathbf{7 0}$ & 60 & 80 & 40 \\
c13-2 & 2 & $\underline{\underline{50}}$ & 60 & $\mathbf{9 0}$ & 70 & 70 & 70 & $\underline{50}$ \\
c19-3 & 3 & $\underline{\underline{20}}$ & 40 & $\mathbf{9 0}$ & 60 & 40 & 70 & $\underline{20}$ \\
c23-3 & 3 & $\underline{20}$ & $\mathbf{7 0}$ & 60 & 60 & 50 & 70 & 60 \\
c32-5 & 5 & $\underline{10}$ & 40 & $\mathbf{6 0}$ & 50 & 30 & 40 & $\underline{10}$ \\
\hline
\end{tabular}


Appendix B. Probabilities elicited from experts in round 2

\begin{tabular}{lllllllll} 
Scenario & $\#$ & E1 & E2 & E3 & E4 & E5 & E6 & E7 \\
\hline c2-1 & 1 & 80 & $\underline{70}$ & $\underline{70}$ & 80 & $\underline{70}$ & $\mathbf{9 0}$ & $\mathbf{9 0}$ \\
c4-1 & 1 & $\underline{50}$ & 70 & $\mathbf{8 0}$ & $\mathbf{8 0}$ & 60 & $\mathbf{8 0}$ & $\mathbf{8 0}$ \\
c8-2 & 2 & 50 & $\mathbf{9 0}$ & 70 & 70 & $\underline{40}$ & 60 & 70 \\
c9-2 & 2 & $\underline{30}$ & $\mathbf{9 0}$ & $\underline{30}$ & 80 & 60 & 70 & 70 \\
c10-2 & 2 & 50 & $\underline{40}$ & 60 & $\mathbf{7 0}$ & 60 & $\mathbf{7 0}$ & $\mathbf{7 0}$ \\
c14-2 & 2 & $\underline{30}$ & 50 & $\mathbf{8 0}$ & 70 & 60 & 50 & 70 \\
c15-2 & 2 & 50 & 50 & 60 & 70 & $\underline{40}$ & 50 & $\mathbf{8 0}$ \\
c17-3 & 3 & $\underline{10}$ & $\mathbf{7 0}$ & 60 & 60 & 30 & 50 & 60 \\
c23-3 & 3 & 40 & $\underline{20}$ & $\underline{20}$ & $\mathbf{7 0}$ & 40 & 30 & $\mathbf{7 0}$ \\
c25-3 & 3 & $\underline{20}$ & $\underline{20}$ & $\mathbf{6 0}$ & $\mathbf{6 0}$ & $\mathbf{6 0}$ & 30 & $\mathbf{6 0}$ \\
$\mathrm{c} 26-3$ & 3 & $\underline{20}$ & $\underline{20}$ & 30 & $\mathbf{7 0}$ & 50 & 50 & 60 \\
c27-4 & 4 & $\underline{10}$ & 40 & 20 & $\mathbf{6 0}$ & 30 & $\mathbf{6 0}$ & 30 \\
$\mathrm{c} 29-4$ & 4 & $\underline{20}$ & 50 & 30 & $\mathbf{6 0}$ & 30 & 40 & 30 \\
$\mathrm{c} 30-4$ & 4 & $\underline{1}$ & 50 & 30 & $\mathbf{6 0}$ & 30 & 50 & 40 \\
\hline
\end{tabular}

Appendix C. Probabilities elicited from experts in round 3

\begin{tabular}{lllllllll}
\hline Scenario & $\#$ & E1 & E2 & E3 & E4 & E5 & E6 & E7 \\
\hline c1-0 & 0 & 90 & 90 & 90 & 90 & $\underline{80}$ & $\mathbf{9 9}$ & $\mathbf{9 9}$ \\
c2-1 & 1 & 80 & 70 & 90 & 80 & $\underline{60}$ & $\mathbf{9 9}$ & 90 \\
c3-1 & 1 & $\underline{30}$ & $\mathbf{9 0}$ & $\mathbf{9 0}$ & 80 & 60 & $\mathbf{9 0}$ & 80 \\
c4-1 & 1 & $\underline{50}$ & 70 & $\mathbf{9 0}$ & 80 & 60 & 80 & 80 \\
c5-1 & 1 & $\mathbf{7 0}$ & 70 & 80 & 80 & $\underline{50}$ & $\mathbf{9 0}$ & 60 \\
c6-1 & 1 & $\underline{30}$ & 60 & $\mathbf{9 0}$ & 80 & 70 & 70 & 70 \\
c7-2 & 2 & $\underline{20}$ & 80 & $\mathbf{9 0}$ & 70 & 50 & 80 & 70 \\
c8-2 & 2 & $\underline{30}$ & $\mathbf{9 0}$ & 80 & 70 & 50 & 70 & 70 \\
c9-2 & 2 & $\underline{20}$ & $\mathbf{9 0}$ & 70 & 80 & 60 & 80 & 50 \\
c10-2 & 2 & $\underline{20}$ & 60 & $\mathbf{8 0}$ & 70 & 60 & $\mathbf{8 0}$ & 70 \\
c11-2 & 2 & $\underline{40}$ & 50 & $\mathbf{8 0}$ & 70 & $\underline{40}$ & 70 & 70 \\
c12-2 & 2 & 60 & 50 & 70 & $\mathbf{8 0}$ & $\underline{30}$ & $\mathbf{8 0}$ & 50 \\
c13-2 & 2 & $\underline{10}$ & $\mathbf{8 0}$ & $\mathbf{8 0}$ & 70 & 60 & 70 & 40 \\
c14-2 & 2 & $\underline{20}$ & 50 & $\mathbf{8 0}$ & 70 & 60 & 60 & 60 \\
c15-2 & 2 & $\underline{50}$ & 40 & $\underline{30}$ & $\mathbf{8 0}$ & 60 & $\mathbf{8 0}$ & 50 \\
c16-2 & 2 & $\underline{20}$ & 40 & $\mathbf{7 0}$ & $\mathbf{7 0}$ & 40 & $\mathbf{7 0}$ & 60 \\
c17-3 & 3 & $\underline{20}$ & $\mathbf{7 0}$ & $\mathbf{7 0}$ & 60 & 30 & 60 & 50 \\
c18-3 & 3 & $\underline{10}$ & $\mathbf{7 0}$ & 60 & $\mathbf{7 0}$ & 40 & $\mathbf{7 0}$ & 40 \\
c19-3 & 3 & $\underline{10}$ & 40 & $\mathbf{7 0}$ & 60 & 50 & $\mathbf{7 0}$ & 50 \\
c20-3 & 3 & $\underline{10}$ & 60 & 60 & $\mathbf{7 0}$ & 50 & $\mathbf{7 0}$ & 30 \\
\hline
\end{tabular}




\begin{tabular}{lllllllll}
\hline Scenario & $\#$ & E1 & E2 & E3 & E4 & E5 & E6 & E7 \\
\hline c21-3 & 3 & $\underline{10}$ & $\mathbf{7 0}$ & 60 & $\mathbf{7 0}$ & 50 & 60 & 30 \\
c22-3 & 3 & $\frac{1}{60}$ & $\mathbf{7 0}$ & $\mathbf{7 0}$ & 30 & 60 & 30 \\
c23-3 & 3 & 40 & $\underline{20}$ & $\underline{20}$ & $\mathbf{7 0}$ & 50 & $\mathbf{7 0}$ & 40 \\
c24-3 & 3 & $\frac{1}{50}$ & 60 & $\mathbf{7 0}$ & 40 & 60 & 20 \\
c25-3 & 3 & $\underline{10}$ & 20 & $\mathbf{7 0}$ & 60 & 40 & 50 & 50 \\
c26-3 & 3 & $\underline{20}$ & 50 & 30 & $\mathbf{7 0}$ & 50 & 30 & 30 \\
c27-4 & 4 & $\underline{1}$ & 40 & $\mathbf{6 0}$ & $\mathbf{6 0}$ & 20 & 60 & 20 \\
c28-4 & 4 & $\underline{1}$ & 50 & 50 & $\mathbf{6 0}$ & 20 & 50 & 20 \\
c29-4 & 4 & $\frac{1}{70}$ & 30 & 60 & 20 & 50 & 20 \\
c30-4 & 4 & $\underline{1}$ & 50 & 40 & $\mathbf{6 0}$ & 50 & 20 & 10 \\
c31-4 & 4 & $\underline{10}$ & 30 & 20 & $\mathbf{6 0}$ & 40 & 20 & 20 \\
c32-5 & 5 & $\underline{1}$ & 30 & 30 & $\mathbf{4 0}$ & 10 & 10 & $\underline{1}$ \\
\hline
\end{tabular}

